\title{
High-Risk Whole Lung Lavage for An Unprecedented Oil Drowning Situation: Management Issues from a Respiratory Therapist's Perspective
}

\author{
Anmol Hanamant Thorbole, Hari Prasad, Sameer Bansal, Ravindra Mehta \\ Department of Pulmonary, Critical Care and Sleep Medicine, Apollo Hospitals, Bengaluru, Karnataka, India
}

\section{Abstract}

Bilateral whole lung lavage (WLL), a complex procedure involving large volume saline washes of both lungs is a standard treatment for pulmonary alveolar proteinosis. Uncommonly, following large volume oil aspiration, the ensuing fulminant lipoid pneumonia ("oil drowning") can be fatal, and therapeutic WLL can be lifesaving. In this report, we describe an oil drowning situation salvaged with high-risk WLL and highlight procedural and critical care aspects from a respiratory therapist's perspective.

Keywords: Acute respiratory distress syndrome, extracorporeal membrane oxygenation, lipoid pneumonia, oil drowning, rescue therapy, rigid bronchoscopy

\section{INTRODUCTION}

Lipoid pneumonia, with its extreme form of large volume oil aspiration (oil drowning) can lead to severe hypoxia and a fatal outcome. Whole lung lavage (WLL) has been used as a salvage therapy in select patients. ${ }^{[1]}$ We present a case of oil drowning and severe hypoxia with respiratory failure who recovered with therapeutic WLL and advanced critical care, focusing on respiratory therapy aspects.

\section{Case Report}

A 31-year-old healthy male was airlifted to our center with a history of accidental fall from a height of $5 \mathrm{~m}$ into an industrial coolant oil tank. He was intubated and mechanically ventilated in view of severe hypoxia. On transfer, vitals included a regular pulse of $80 / \mathrm{min}$, blood pressure $130 / 90$ $\mathrm{mm} \mathrm{Hg}$, and oxygen saturation $\left(\mathrm{SpO}_{2}\right)$ of $90 \%$ on ventilator support with an inspiratory fraction of oxygen $\left(\mathrm{FiO}_{2}\right)$ of 1.0. Laboratory tests were within normal limits. Computerized tomography scan of the thorax showed bilateral ground-glass opacities (GGO) with a crazy-paving appearance [Figure 1]. Emergent bedside bronchoscopy showed thick oily sludge, with purulent secretions and thick plugs blocking the airways. Bronchoalveolar lavage (BAL) cytology demonstrated the

\begin{tabular}{|l|l|}
\hline \multicolumn{3}{|c|}{ Access this article online } \\
\hline Quick Response Code: & Website: \\
\hline & www.ijrc.in \\
\hline
\end{tabular}

presence of extensive fat globules on oil red-O staining. The clinical picture and imaging were consistent with exogenous lipoid pneumonia secondary to massive oil aspiration, with an additional mechanical component of oil obstruction with impaired mucociliary clearance. Broad-spectrum antibiotics were continued. In view of severe hypoxemia and the bronchoscopy findings, WLL was required. Severe baseline hypoxemia precluded a conventional WLL and hence WLL with veno-venous extracorporeal membrane oxygenation (ECMO) support was planned. After ECMO was established, the patient was intubated with a rigid bronchoscope to facilitate clearance of the viscous oily sludge [Figure 2]. The right lung was lavaged with $15 \mathrm{~L}$ and the left lung with $10 \mathrm{~L}$ of pyrogen-free saline. Postprocedure patient was re-intubated with an endotracheal tube (ETT) and shifted to the intensive care unit. In the next 2 days, there was a significant improvement

Address for correspondence: Ms. Anmol Thorbole, Department of Pulmonary Critical Care and Sleep Medicine, Apollo Hospitals, Bangalore,India. E-mail: anmolthorbole24@gmail.com

This is an open access journal, and articles are distributed under the terms of the Creative Commons Attribution-NonCommercial-ShareAlike 4.0 License, which allows others to remix, tweak, and build upon the work non-commercially, as long as appropriate credit is given and the new creations are licensed under the identical terms.

For reprints contact: WKHLRPMedknow_reprints@wolterskluwer.com

How to cite this article: Thorbole $\mathrm{AH}$, Prasad H, Bansal S, Mehta R. High-risk whole lung lavage for an unprecedented oil drowning situation: Management issues from a respiratory therapist's perspective. Indian J Respir Care 2021;10:260-2.

Received: $13-03-2021$ Accepted: $23-05-2021$

Revised: $02-05-2021$ Published: 14-06-2021 
in oxygenation and the ECMO support was removed. Critical care issues involved suctioning the remnants of viscid fluid post-WLL. He was weaned over the next 2-3 days, extubated to high flow nasal oxygen, and discharged on day 15 with a $\mathrm{SpO}_{2}$ of $96 \%$ room air. The chest X-ray postextubation showed significant improvement in GGO [Figure 3].

Specific issues to management by respiratory therapist (RT) include the following:

1. Initial ventilation was a challenge due to mechanical blockage with thick viscid secretions and required a lower tidal volume, higher respiratory rate, and minute

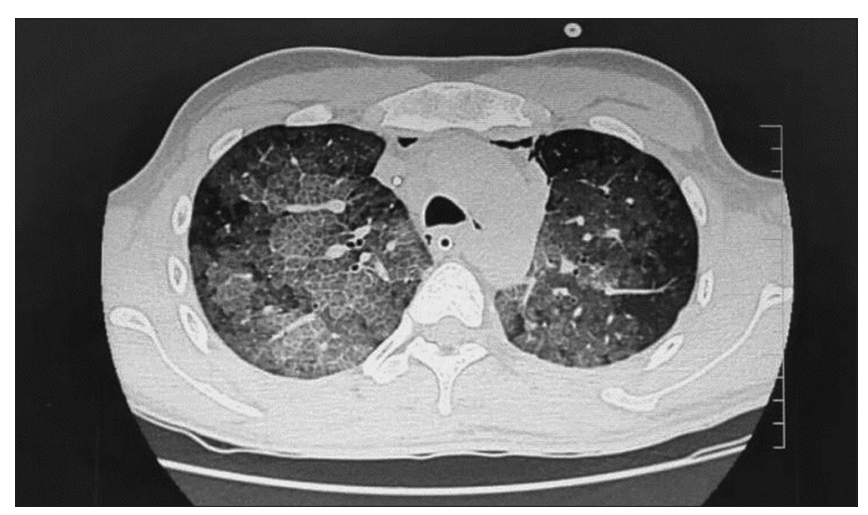

Figure 1: Computerized tomography thorax showing crazy-paving pattern

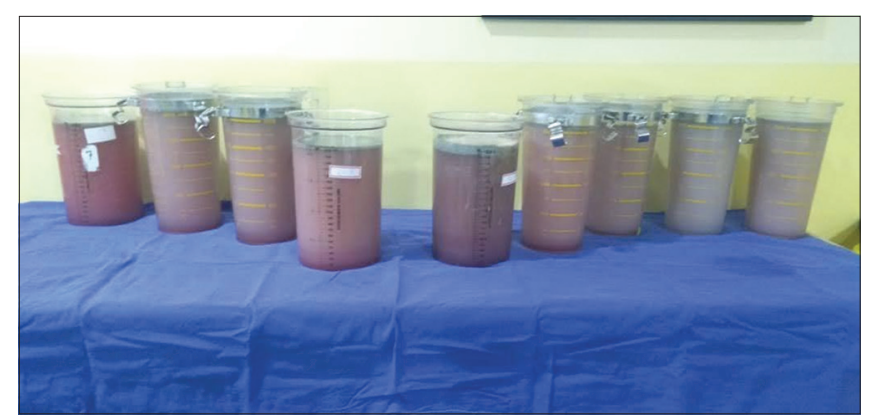

Figure 2: Whole lung lavage return fluid with oil floating on the top

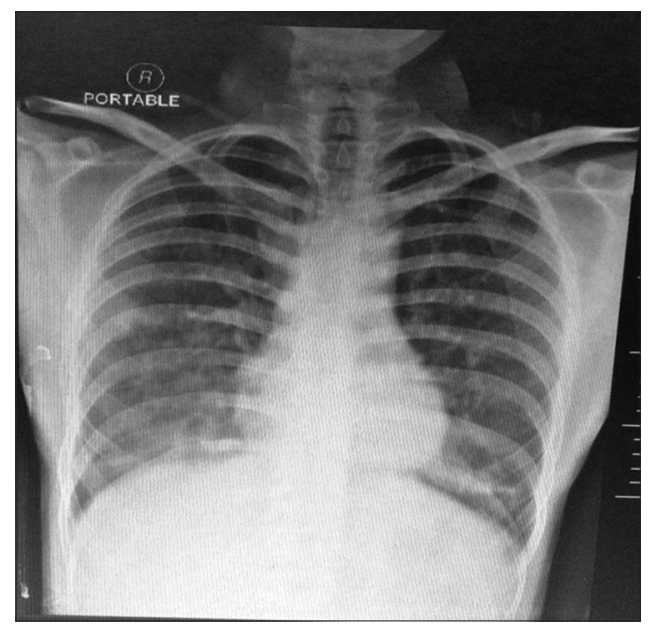

Figure 3: Chest X-ray-posteroanterior view showing clearing post-whole lung lavage ventilation to maintain gas exchange. In addition, wide bore catheters were required for suctioning the oily purulent secretions

2. The initial bronchoscopy assisted by the RT also was complicated due to baseline severe hypoxemia and difficulty suctioning and required repeated runs and flushes to get secretions out - close collaboration between the RT and bronchoscopist was needed

3. Modifications to the WLL technique included percussion and RT-guided position change to both mechanically dislodge the viscid oil and facilitate gravity drainage as adjuncts to the washing

4. Postprocedure ventilator care involved frequent suctioning to clear out the leftover fluid, active secretion mobilization with percussion and suctioning, cautious extubation to high flow nasal cannula, and later weaning to $\mathrm{O}_{2}$. A cough assist device (Philips Mechanical Insufflator-Exsufflator ${ }^{\mathrm{TM}}$ ) was used for augmenting the weak cough reflex and to prevent reintubation.

\section{Discussion}

Lipoid pneumonia is categorized into two groups: exogenous lipoid pneumonia and endogenous lipoid pneumonia. Exogenous lipoid pneumonia is a common form of lipoid pneumonia caused due to aspiration or inhalation of lipids, the extreme form of which is oil drowning. ${ }^{[1]}$ Lipoid pneumonia is usually diagnosed from BAL with fat globules visualized with Oil red-O and Papanicolaou stains. The mainstay of treatment in exogenous lipoid pneumonia is to avoid exposure and provide supportive care ${ }^{[2,3]}$ In extreme cases, there are reports of WLL done in the pediatric age group. ${ }^{[4]}$ Our case describes the challenges faced in the management of adult massive oil aspiration, or oil drowning, specifically airway management and severe hypoxemia. Postintubation, the patient continued to have severe hypoxemia secondary to significant oil residue in the airways and consequent pneumonitis/infection and acute respiratory distress syndrome. Despite an inspired $\left(\mathrm{FiO}_{2}\right.$ of 1.0) and positive end-expiratory pressure (PEEP) of $10 \mathrm{~cm} \mathrm{H}_{2} \mathrm{O}$, his $\mathrm{SpO}_{2}$ barely crossed $90 \%$. Aggressive chest percussion with a mechanical vibrator device and frequent ETT suctioning failed to clear the thick oily residue. The oily residue in the airways also led to high airway pressures and difficulty in ventilating the patient. An attempt was made to improve oxygenation by utilizing airway pressure release ventilation mode of ventilation. Prone positioning was also tried but failed to create any measurable change in the respiratory or ventilator parameters. This could have been partly due to the obliteration of the alveolar surfaces by the dense oily material. The presence of oily material imparts an inelastic fibrotic component to the lungs, and makes it resistant to recruitment by means of PEEP or prone positioning.

WLL was the only means to salvage the patient in view of steadily worsening hypoxia. WLL in lipoid pneumonia has been described in a few cases worldwide, in cases where there is an 
acute aspiration of massive amounts of oil. ${ }^{[5]}$ Our "oil drowning" case represented the critical end of the lipoid pneumonia spectrum which required WLL with ECMO support. A unique aspect of our case was the first of its kind use of a rigid bronchoscope for WLL, wherein the barrel of the rigid bronchoscope was used as a conduit for introducing large-bore suction devices. To the best of our knowledge, this technique has never been attempted for WLL in lipoid pneumonia. This was made possible because the patient's oxygenation was maintained with ECMO while the lung lavage could be carried out unhindered using a rigid bronchoscope. At all stages from initial ventilation, WLL procedure to post-WLL critical care, RT involvement as mentioned above was an important part of the interdisciplinary team.

\section{ConcLusion}

Oil drowning, the most severe form of lipoid pneumonia can be catastrophic and needs salvage measures, in this case WLL supported by ECMO. This interesting case highlights important RT aspects at all stages of management of this uncommon but life-threatening condition.

\section{Declaration of patient consent}

The authors certify that they have obtained all appropriate patient consent forms. In the form the patient(s) has/have given his/her/their consent for his/her/their images and other clinical information to be reported in the journal. The patients understand that their names and initials will not be published and due efforts will be made to conceal their identity, but anonymity cannot be guaranteed.

\section{Financial support and sponsorship}

Nil.

\section{Conflicts of interest}

There are no conflicts of interest.

\section{RefEREnCes}

1. Indira KS, Rajesh V, Darsana V, Ranjit U, John J, Vengadakrishnaraj SP, et al. Whole lung lavage: The salvage therapy for pulmonary alveolar proteinosis. Indian J Chest Dis Allied Sci 2007;49:41-4.

2. Laughlen GF. Studies on pneumonia following nasopharyngeal injection of oil. Am J Pathol 1925;1:407-14.

3. Spickard A $3^{\text {rd }}$, Hirschmann JV. Exogenous lipoid pneumonia. Arch Intern Med 1994;154:686-92.

4. Ciravegna B, Sacco O, Moroni C, Silvestri M, Pallecchi A, Loy A, et al. Mineral oil lipoid pneumonia in a child with anoxic encephalopathy: Treatment by whole lung lavage. Pediatr Pulmonol 1997;23:233-7.

5. Sias SM, Ferreira AS, Daltro PA, Caetano RL, Moreira Jda S, Quirico-Santos T. Evolution of exogenous lipoid pneumonia in children: Clinical aspects, radiological aspects and the role of bronchoalveolar lavage. J Bras Pneumol 2009;35:839-45. 